\title{
深空探测器的双曲线轨道及其变化规律 ${ }^{*}$
}

\author{
刘 林 王 敦 \\ (南京大学天文系, 南京 210093)
}

\begin{abstract}
摘要深空探测器在接近目标天体而未变轨前的运行段, 相对目标天体而言通常是 双曲线轨道. 针对这一特点, 类似研究受摄椭圆轨道那样, 给出双曲线轨道在各种摄动 力影响下的变化规律, 为该运行段的星历计算(特别是星上轨道外推)、变轨和轨控提供 一种分析型的计算公式或相关轨道信息.
\end{abstract}

\section{关键词深空探测器 双曲线轨道 受摄运动}

作为一个力学系统, 太阳系中各种大小天体的运动, 基本上都对应一个变化的椭圆轨道 或一般周期轨道(或拟周期轨道). 因此, 在天体力学和卫星轨道力学所涉及的书籍和有关文章 中, 很少提及双曲线轨道, 更不会讨论在受摄情况下这种轨道的变化规律, 因为这种轨道对应 的天体将要飞离它所在的系统. 但是, 对于深空探测器的运动而言, 当它从地球停泊轨道上飞 抵目标天体附近时, 在未变轨前, 相对目标天体的运动通常是双曲线轨道. 要使其变为绕目标 天体运行的轨道器, 就必须再次变轨, 将双曲线轨道变为椭圆(或近圆)轨道; 或者仅需接近探 测过程中的某个目标天体, 绕飞或接近该目标天体后离去. 无论是前一种探测形式, 还是后一 种探测器接近目标天体时, 相对该天体总有一段运行轨道是双曲线轨道. 为了更好地了解探 测器的运行状态, 给地面测控系统或是星上自主设备提供必要的轨道信息(而不仅仅是探测器 在一些孤立点上的位置和速度), 有必要对双曲线轨道的变化特征进行研究, 给出类似于椭圆 轨道变化的一些基本规律. 本文在双曲线运动 6 个积分 ${ }^{[14]}$ 的基础上给出了受摄双曲线运动对 应的摄动运动方程, 并以目标天体的扁率摄动为例, 导出了相应的轨道变化的近似分析解, 并 以月球探测器为例与精确的数值解作了比对.

\section{1 双曲线轨道的 6 个积分和基本关系式}

作为探测器相对目标天体的运动, 双曲线轨道是相应二体问题解的 1 种形式. 在目标天体 的质心赤道坐标系中, 探测器的相对运动满足下列微分方程:

$$
\ddot{r}=-\frac{\mu}{r^{3}} \boldsymbol{r}
$$

其中 $\mu=G M_{0}, G$ 是万有引力常数, $M_{0}$ 是目标天体的质量(这里当作一个质点处理), $\boldsymbol{r}, \dot{\boldsymbol{r}}, \ddot{\boldsymbol{r}}$ 各为 探测器的位置、速度和加速度矢量. 该问题的 6 个积分 ${ }^{[1 \sim 4]}$ 如下:

$$
\begin{gathered}
\boldsymbol{r} \times \dot{\boldsymbol{r}}=\boldsymbol{h}=h \hat{R}, \\
h=|\boldsymbol{r} \times \dot{\boldsymbol{r}}|,
\end{gathered}
$$




$$
\begin{gathered}
\hat{R}=(\boldsymbol{r} \times \dot{\boldsymbol{r}}) / h=\left(\begin{array}{c}
\sin i \sin \Omega \\
-\sin i \cos \Omega \\
\cos i
\end{array}\right), \\
r=\frac{a\left(e^{2}-1\right)}{1+e \cos (\theta-\omega)}=\frac{p}{1+e \cos f}, \\
h=\sqrt{\mu p}, \quad p=a\left(e^{2}-1\right), \\
e \operatorname{sh} E-E=n(t-\tau)=M, \quad n=\sqrt{\mu} a^{-3 / 2},
\end{gathered}
$$

轨道积分中 $f=\theta-\omega$ 是真近点角, 其变化范围是 $\left(-\pi+\cos ^{-1}\left(\frac{1}{e}\right), \pi-\cos ^{-1}\left(\frac{1}{e}\right)\right), E$ 是描述双曲线的 一个参数, 作用类似于椭圆中的偏近点角. 这里 $a, e, i . \Omega, \omega, \tau$ (或 $M) 6$ 个积分常数即双曲线轨道 的 6 个轨道根数 (记为 $\sigma$ ), 其意义与椭圆轨道的 6 个根数类似, 这里 $e>1$.

相应的一些基本关系公式有

$$
\begin{gathered}
v^{2}=\mu\left(\frac{2}{r}+\frac{1}{a}\right), \quad v^{2}=|\dot{r}|^{2}, \\
\qquad \begin{array}{c}
r=a(e \operatorname{ch} E-1), \\
r \cos f=a(e-\operatorname{ch} E) \\
r \sin f=a \sqrt{e^{2}-1} \operatorname{sh} E
\end{array}, \quad \tan \frac{f}{2}=\sqrt{\frac{e+1}{e-1}} \operatorname{th} \frac{E}{2} .
\end{gathered}
$$

\section{2 位置速度矢量 $(\boldsymbol{r}, \dot{\boldsymbol{r}})$ 与轨道根数 $\boldsymbol{\sigma}$ 之间的关系}

( i ) 星历计算 $\sigma \Rightarrow(\boldsymbol{r}, \dot{\boldsymbol{r}})$

$$
\left\{\begin{array}{l}
\boldsymbol{r}=r \cos f \hat{P}+r \sin f \hat{Q}, \\
\dot{\boldsymbol{r}}=\sqrt{\frac{\mu}{p}}[(-\sin f) \hat{P}+(\cos f+e) \hat{Q}]
\end{array}\right.
$$

或

$$
\left\{\begin{array}{l}
\boldsymbol{r}=a(e-\operatorname{ch} E) \hat{P}+a \sqrt{e^{2}-1} \operatorname{sh} E \hat{Q}, \\
\dot{\boldsymbol{r}}=\frac{\sqrt{\mu a}}{r}\left[(-\operatorname{sh} E) \hat{P}+\left(\sqrt{e^{2}-1} \operatorname{ch} E\right) \hat{Q}\right],
\end{array}\right.
$$

这里同样涉及到由 “Kepler” 积分(7)式求解 $E$ 的问题, 不妨称(7)式为广义 Kepler 方程, 其解 法与椭圆轨道中 Kepler 方程的解法有类似之处, 但由于 $e>1$, 不能采用简单的迭代法. 两个 单位矢量 $\hat{P}$ 和 $\hat{Q}$ 是轨道根数 $i, \Omega, \omega$ 的函数, 其具体表达式与椭圆轨道完全相同.

(ii) 轨道计算中的 $(\boldsymbol{r}, \dot{\boldsymbol{r}}) \Rightarrow \sigma$

由下列各式分别计算 $a, e, E, M$ :

$$
\begin{gathered}
\frac{1}{a}=\frac{v^{2}}{\mu}-\frac{2}{r}, \\
\left\{\begin{array}{l}
e \operatorname{sh} E=(r \dot{r}) / \sqrt{\mu a}, \\
e \operatorname{ch} E=\left(\frac{r}{a}\right)+1 .
\end{array}\right.
\end{gathered}
$$




$$
\left\{\begin{array}{c}
e^{2}=(e \operatorname{ch} E)^{2}-(e \operatorname{sh} E)^{2}, \\
E=\operatorname{Arth} x=\frac{1}{2} \ln \left(\frac{1+x}{1-x}\right), \quad x=\frac{e \operatorname{sh} E}{e \operatorname{ch} E}, \\
M=e \operatorname{sh} E-E .
\end{array}\right.
$$

关于 $i, \Omega, \omega$ 的计算，与椭圆轨道中的计算基本相同，有

$$
\begin{gathered}
\cos i=(\boldsymbol{r} \times \dot{\boldsymbol{r}})_{z} / \sqrt{\mu p}, \\
\left\{\begin{array}{l}
\sin i \sin \Omega=(\boldsymbol{r} \times \dot{\boldsymbol{r}})_{x} / \sqrt{\mu p}, \\
\sin i \cos \Omega=-(\boldsymbol{r} \times \dot{\boldsymbol{r}})_{y} / \sqrt{\mu p},
\end{array}\right. \\
\left\{\begin{array}{l}
\sin i \sin \omega=P_{z}, \\
\sin i \cos \omega=Q_{z},
\end{array}\right.
\end{gathered}
$$

其中

$$
\left\{\begin{array}{l}
P_{z}=(\hat{P})_{z}, \quad Q_{z}=(\hat{Q})_{z}, \\
\hat{P}=\left(\frac{\operatorname{ch} E}{r}\right) \boldsymbol{r}-\left(\frac{\sqrt{a}}{\sqrt{\mu}} \operatorname{sh} E\right) \dot{\boldsymbol{r}}, \\
\hat{Q}=\frac{1}{\sqrt{e^{2}-1}}\left[\left(\frac{\operatorname{sh} E}{r}\right) \boldsymbol{r}+\frac{\sqrt{a}}{\sqrt{\mu}}(e-\operatorname{ch} E) \dot{\boldsymbol{r}}\right] .
\end{array}\right.
$$

\section{3 受摄双曲线运动的摄动运动方程}

双曲线运动的上述 6 个积分可写成如下形式:

$$
\varphi(a, e, i, \Omega, \omega, \tau)=\psi(\boldsymbol{r}, \dot{\boldsymbol{r}})
$$

受摄运动方程的一般形式为

$$
\left\{\begin{aligned}
\ddot{\boldsymbol{r}} & =\boldsymbol{F}_{0}(\boldsymbol{r})+\boldsymbol{F}_{\varepsilon}(\boldsymbol{r}, \dot{\boldsymbol{r}}, t ; \varepsilon), \\
\boldsymbol{F}_{0} & =-\frac{\mu}{r^{3}} \boldsymbol{r} .
\end{aligned}\right.
$$

根据常数变易原理可给出

$$
\sum_{j=1}^{6} \frac{\partial \varphi}{\partial \sigma_{j}} \dot{\sigma}_{j}=\left(\frac{\partial \psi}{\partial \dot{\boldsymbol{r}}}\right)^{T} \cdot \boldsymbol{F}_{\varepsilon}
$$

或分别写成下列形式:

$$
\sum_{j=1}^{6} \frac{\partial \varphi}{\partial \sigma_{j}} \dot{\sigma}_{j}= \begin{cases}\frac{\partial \psi}{\partial \dot{x}}\left(F_{\varepsilon}\right)_{x}+\frac{\partial \psi}{\partial \dot{y}}\left(F_{\varepsilon}\right)_{y}+\frac{\partial \psi}{\partial \dot{z}}\left(F_{\varepsilon}\right)_{z} & \text { 直角坐标系, } \\ \frac{\partial \psi}{\partial \dot{r}} S+\frac{\partial \psi}{r \partial \dot{\theta}} T+\frac{\partial \psi}{\partial \dot{z}} W & \text { 空间极坐标系, }\end{cases}
$$

其中 $S, T, W$ 分别为摄动加速度 $\boldsymbol{F}_{\mathcal{E}}$ 的径向、横向和轨道面法向分量.

利用常数变易关系(24)式, 略去推导过程直接写出双曲线轨道变化所满足的摄动运动方 程如下: 


$$
\begin{aligned}
\frac{\mathrm{d} a}{\mathrm{~d} t} & =-\frac{2}{n \sqrt{e^{2}-1}}[(e \sin f) S+(1+e \cos f) T], \\
\frac{\mathrm{d} e}{\mathrm{~d} t} & =\frac{\sqrt{e^{2}-1}}{n a}[(\sin f) S+(\cos f+\operatorname{ch} E) T], \\
\frac{\mathrm{d} i}{\mathrm{~d} t} & =\frac{r \cos u}{n a^{2} \sqrt{e^{2}-1}} W, \\
\frac{\mathrm{d} \Omega}{\mathrm{d} t} & =\frac{r \sin u}{n a^{2} \sqrt{e^{2}-1} \sin i} W, \\
\frac{\mathrm{d} \omega}{\mathrm{d} t} & =\frac{\sqrt{e^{2}-1}}{n a e}\left[(-\cos f) S+\sin f\left(1+\frac{r}{p}\right) T\right]-\cos i \frac{\mathrm{d} \Omega}{\mathrm{d} t}, \\
\frac{\mathrm{d} M}{\mathrm{~d} t} & =n+\frac{e^{2}-1}{n a e}\left[\left(-\cos f+2 e \frac{r}{p}\right) S+\sin f\left(1+\frac{r}{p}\right) T\right]
\end{aligned}
$$

或

$$
\begin{aligned}
\frac{\mathrm{d} a}{\mathrm{~d} t} & =-\frac{2}{n a}\left(\frac{\partial R}{\partial M}\right), \\
\frac{\mathrm{d} e}{\mathrm{~d} t} & =\frac{\left(e^{2}-1\right)}{n a^{2} e}\left(\frac{\partial R}{\partial M}\right)+\frac{\sqrt{e^{2}-1}}{n a^{2} e}\left(\frac{\partial R}{\partial \omega}\right), \\
\frac{\mathrm{d} i}{\mathrm{~d} t} & =\frac{1}{n a^{2} \sqrt{e^{2}-1} \sin i}\left[\cos i\left(\frac{\partial R}{\partial \omega}\right)-\left(\frac{\partial R}{\partial \Omega}\right)\right], \\
\frac{\mathrm{d} \Omega}{\mathrm{d} t} & =\frac{1}{n a^{2} \sqrt{e^{2}-1} \sin i}\left(\frac{\partial R}{\partial i}\right), \\
\frac{\mathrm{d} \omega}{\mathrm{d} t} & =-\frac{\sqrt{e^{2}-1}}{n a^{2} e}\left(\frac{\partial R}{\partial e}\right)-\cos i \frac{\mathrm{d} \Omega}{\mathrm{d} t}, \\
\frac{\mathrm{d} M}{\mathrm{~d} t} & =n-\frac{e^{2}-1}{n a^{2} e}\left(\frac{\partial R}{\partial e}\right)+\frac{2}{n a}\left(\frac{\partial R}{\partial a}\right),
\end{aligned}
$$

其中 $R=\operatorname{grad} \boldsymbol{F}_{\varepsilon}$ 是保守力型摄动对应的摄动函数.

从上述摄动运动方程不难看出, 由于双曲线轨道对应的 $e>1$, 相应的 $a$ 和 $e$ 以及相关的平 近点角 $M$ 的变化规律有别于椭圆轨道. 例如受到阻力摄动时, $a$ 是增大, 注意, 抛物线轨道对 应的 $a \rightarrow \infty, a$ 增大意味着速度减小, 双曲线轨道向抛物线轨道退化. 根据摄动运动方程就可 分析双曲线轨道的某些变化特征.

\section{4 目标天体(中心天体)的扁率摄动}

作为受摄双曲线轨道变化的一个实例, 这里讨论探测器接近目标天体时, 该天体扁率摄 动下的变化规律, 导出相应的摄动分析解, 借此让读者了解构造相应于双曲轨道变化的小参 数幂级数解的具体方法, 同时也可为有关工作提供具体的轨道摄动外推公式.

仍采用标准计算单位，即长度、质量和时间单位分别为 
在此计算单位系统中, $G=1, \mu=G M_{0}=1$.

$$
\left\{\begin{array}{l}
{[L]=R_{e} \text { (中心天体赤道半径), }} \\
{[M]=M_{0} \text { (中心天体质量), }} \\
{[T]=\left(R_{e}^{3} / G M_{0}\right)^{1 / 2} .}
\end{array}\right.
$$

扁率 $\left(J_{2}\right)$ 项对应的摄动函数为

$$
\begin{aligned}
R & =-\frac{J_{2}}{r^{3}}\left(\frac{3}{2} \sin ^{2} \varphi-\frac{1}{2}\right) \\
& =\frac{J_{2}}{2 a^{3}}\left(\frac{a}{r}\right)^{3}\left[\left(1-\frac{3}{2} \sin ^{2} i\right)+\frac{3}{2} \sin ^{2} i \cos 2 u\right],
\end{aligned}
$$

其中 $u=f+\omega$. 相应的 3 个摄动加速度分量为

$$
\begin{gathered}
S=\frac{\partial R}{\partial r}=-\frac{3 J_{2}}{2 a^{4}}\left(\frac{a}{r}\right)^{4}\left[\left(1-\frac{3}{2} \sin ^{2} i\right)+\frac{3}{2} \sin ^{2} i \cos 2(f+\omega)\right], \\
T=\frac{1}{r} \frac{\partial R}{\partial u}=-\frac{3 J_{2}}{2 a^{4}}\left(\frac{a}{r}\right)^{4}\left[\sin ^{2} i \sin 2(f+\omega)\right], \\
W=\frac{1}{r \sin u} \frac{\partial R}{\partial i}=-\frac{3 J_{2}}{2 a^{4}}\left(\frac{a}{r}\right)^{4}[\sin 2 i \sin (f+\omega)] .
\end{gathered}
$$

将 $S, T, W$ 分别代人摄动运动方程(25)的相应部分即可得轨道变化对应的小参数方程:

$$
\dot{\sigma}=f(\sigma, t ; \varepsilon) \text {. }
$$

由于双曲线轨道是 “开放” 的，近点角 $f$ 和 $M$ 的变化不同于椭圆轨道，故相应摄动解无长 期项和周期项之分, 因此可将轨道解记为

$$
\sigma(t)=\sigma\left(t_{0}\right)+\Delta \sigma(t),
$$

根据解的特征，这里的 $\Delta \sigma(t)$ 又记为下列形式:

$$
\Delta \sigma=\sigma_{s}(t)-\sigma_{s}\left(t_{0}\right),
$$

式中 $\sigma_{s}(t)$ 的一阶形式如下 (略去推导过程):

$$
\begin{gathered}
a_{s}(t)=-\frac{3 J_{2}}{2 a}\left(\frac{a}{r}\right)^{3}\left[\frac{2}{3}\left(1-\frac{3}{2} \sin ^{2} i\right)+\sin ^{2} i \cos (2 f+2 \omega)\right], \\
e_{s}(t)=\frac{3 J_{2}}{2 p^{2}}\left\{\left(1-\frac{3}{2} \sin ^{2} i\right)\left[\left(1+\frac{e^{2}}{4}\right) \cos f+\frac{e}{2} \cos 2 f+\frac{e^{2}}{12} \cos 3 f\right]\right. \\
+\sin ^{2} i\left[\frac{1}{16} e^{2} \cos (f-2 \omega)+\left(\frac{1}{4}+\frac{11}{16} e^{2}\right) \cos (f+2 \omega)+\frac{5}{4} e \cos (2 f+2 \omega)\right. \\
\left.\left.+\left(\frac{7}{12}+\frac{17}{48} e^{2}\right) \cos (3 f+2 \omega)+\frac{3}{8} e \cos (4 f+2 \omega)+\frac{1}{16} e^{2} \cos (5 f+2 \omega)\right]\right\}, \\
i_{s}(t)=\frac{3 J_{2}}{2 p^{2}}\left(\frac{1}{2} \sin 2 i\right)\left[\frac{e}{2} \cos (f+2 \omega)+\frac{1}{2} \cos (2 f+2 \omega)+\frac{e}{6} \cos (3 f+2 \omega)\right], \\
\Omega_{s}(t)=-\frac{3 J_{2}}{2 p^{2}} \cos i\left[(f+e \sin f)-\frac{e}{2} \sin (f+2 \omega)-\frac{1}{2} \sin (2 f+2 \omega)-\frac{e}{6} \sin (3 f+2 \omega)\right],
\end{gathered}
$$




$$
\begin{aligned}
\omega_{s}(t)= & -\cos i \Omega_{s}(t) \\
& +\frac{3 J_{2}}{2 p^{2}}\left\{\left(1-\frac{3}{2} \sin ^{2} i\right)\left[f+\left(\frac{1}{e}+\frac{3}{4} e\right) \sin f+\frac{1}{2} \sin 2 f+\frac{e}{12} \sin 3 f\right]\right. \\
& +\sin ^{2} i\left[\frac{1}{16} e \sin (f-2 \omega)-\left(\frac{1}{4 e}-\frac{7}{16} e\right) \sin (f+2 \omega)+\frac{3}{4} \sin (2 f+2 \omega)\right. \\
& \left.\left.+\left(\frac{7}{12 e}+\frac{11}{48} e\right) \sin (3 f+2 \omega)+\frac{3}{8} \sin (4 f+2 \omega)+\frac{1}{16} e \sin (5 f+2 \omega)\right]\right\}, \\
M_{s}(t)= & \frac{3}{2 a} a_{s}\left(t_{0}\right) M \\
& +\frac{3 J_{2}}{2 p^{2}} \sqrt{e^{2}-1}\left\{\left(1-\frac{3}{2} \sin ^{2} i\right)\left[\left(\frac{1}{e}-\frac{1}{4} e\right) \sin f+\frac{1}{2} \sin 2 f+\frac{e}{12} \sin 3 f\right]\right. \\
& +\sin ^{2} i\left[\frac{1}{16} e \sin (f-2 \omega)-\left(\frac{1}{4 e}+\frac{5}{16} e\right) \sin (f+2 \omega)\right. \\
& \left.\left.+\left(\frac{7}{12 e}-\frac{1}{48} e\right) \sin (3 f+2 \omega)+\frac{3}{8} \sin (4 f+2 \omega)+\frac{1}{16} e \sin (5 f+2 \omega)\right]\right\} .
\end{aligned}
$$

上述各式中 $a=a_{0}, e=e_{0}, i=i_{0}, \Omega=\Omega_{0}, \omega=\omega_{0}, M=M(t), M_{0}=M\left(t_{0}\right)$.

\section{5 计算比对-摄动分析解的精度}

以月球探测器为例, 探测器飞抵月球作用范围边界时, 相对月球为双曲线运动. 作为算例, 我们选取了向月飞行轨道的一种, 即探测器到达该作用范围边界(月心距为 $57800 \mathrm{~km}=33.26 R_{e}$, $R_{e}=1738 \mathrm{~km}$ 是月球赤道半径)时, 相对月球的速度为 $0.903783403 \mathrm{~km} / \mathrm{s}=0.538105019$, 相应 的近月点高度为 $100 \mathrm{~km}$. 这一双曲线轨道(这里取顺行轨道, 对逆行轨道, 计算情况相同)的 6 个初始根数为

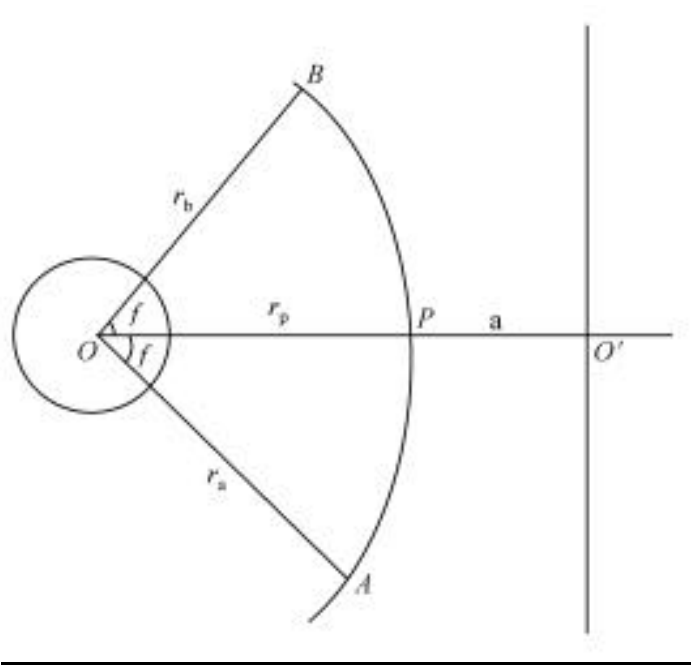

图 1 双曲线轨道

$$
\begin{cases}a_{0}=4.358728198, & \Omega_{0}=45^{\circ}, \\ e_{0}=1.242625222, & \omega_{0}=45^{\circ}, \\ i_{0}=45^{\circ}, & M_{0}=-338^{\circ} .88866330 .\end{cases}
$$

我们分别用高精度数值解(积分器为 RKF7 (8) ${ }^{[5]}$ ) 和本文给出的摄动分析解计算探测器从进人月 球作用范围(图 1 中的 $A$ 点)到飞离作用范围(图 1 中的 $B$ 点)这一关键弧段上, 双曲线轨道的变 化. 按二体问题，这一弧段的飞行时间为 $1^{\mathrm{d}} .2892668=1856^{\mathrm{m}} .544192$, 计算结果列于表 1 .

从计算结果可以看出, 以数值解为标准, 分析解的误差也只有 $10^{-8}$, 这与本文给出的一 阶摄动解的精度是相当的, 在这一弧段上, 月 球扁率摄动量级为 $10^{-5} \sim 10^{-4}$, 而又是保守力 摄动, 二阶摄动的量级应为 $10^{-8}$. 对于探测器的运动, 另一种重要摄动源是第 3 体(地球)的引 
表 1 两种方法给出的根数和坐标速度矢量的结果

\begin{tabular}{cccccc}
\hline 根数 & 数值法 & 分析法 & 坐标速度 & 数值解 & 分析解 \\
\hline$a\left(R_{e}\right)$ & 4.358728041280 & 4.358728041277 & $x\left(R_{e}\right)$ & -22.5037946917 & -22.5037946623 \\
$E$ & 1.242623185315 & 1.242623181860 & $y\left(R_{e}\right)$ & -24.4517219010 & -24.4517219719 \\
$i /\left(^{\circ}\right)$ & 44.9997324696 & 44.9997320198 & $z\left(R_{e}\right)$ & -1.3856476350 & -1.3856470591 \\
$\Omega /\left(^{\circ}\right)$ & 44.9857322459 & 44.9857333244 & $\dot{x} / \mathrm{km} \cdot \mathrm{s}^{-1}$ & -0.5671864971 & -0.5671864968 \\
$\omega /\left(^{\circ}\right)$ & 45.0150039744 & 45.0150023362 & $\dot{y} / \mathrm{km} \cdot \mathrm{s}^{-1}$ & -0.6975563433 & -0.6975563457 \\
$M /\left(^{\circ}\right)$ & 338.8885835763 & 338.8885835407 & $\dot{z} / \mathrm{km} \cdot \mathrm{s}^{-1}$ & -0.0924072365 & -0.0924072201 \\
\hline
\end{tabular}

力摄动, 但其影响不会超过月球扁率摄动. 故本文给出的双曲线轨道的一阶摄动分析解确有 实际意义，而建立第 3 体引力摄动并不困难，本文不再列出.

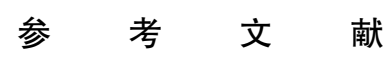

1 易照华. 天体力学基础. 南京: 南京大学出版社, 1997. $32 \sim 34$

2 刘 林. 天体力学方法. 南京: 南京大学出版社, 1998. 37 39

3 Taff L G. Celestial Mechanics. New York: John Wiley \& Sons. 1985. $45 \sim 47$

4 Battin R H. An Introduction to the Mathematics and Methods of Astrodynamics. New York: AIAA Education Series, 1987. $165 \sim 173$

5 Fehlberg E. Classical fifth- sixth- seventh and eighth order Runge-Kutta formulas with stepsize control. NASA TR R-287, 1968 